\title{
473989 - RETROSPECTIVE AUDIT OF PAIN MANAGEMENT FOLLOWING ANTERIOR RESECTION
}

\author{
Natasha Broemling, BSc., MD. ${ }^{1}$, Penny Brasher, PhD $^{2}$, Kelly Mayson, MD, FRCPC ${ }^{1}$ \\ 1. Anesthesiology, Pharmacology \& Therapeutics, University of British Columbia, \\ Vancouver, BC, Canada \\ 2. Centre for Clinical Epidemiology \& Evaluation, Vancouver Coastal Health \\ Research Institute, Vancouver, BC, Canada
}

Introduction: We conducted an observational study to review anesthetic pain management and corresponding patient outcomes following anterior resection surgery. A pilot audit suggested a high incidence of excessive pain in the recovery room following this procedure. We hypothesized that multimodal pain management was not used in the majority of these patients, and secondly, that differences existed in the care practices of the anesthesiologists whose patients had excessive pain in the recovery room, compared to those whose patients did not.

Methods: At our tertiary care centre, a computerized surgical patient tracking system links booked procedures with complications in the recovery room, including excessive pain. PACU nurses identify excessive pain as an inability to decrease pain intensity to a level acceptable to the patient, or nursing care dominated by pain management. After institutional review board approval, a retrospective chart audit was performed. 109 patients were found to have undergone anterior resections between April 2002 and March 2006. Patients with excessive pain were compared to the remaining patients. Baseline population characteristics, pain interventions and end-points were collected. Multimodal analgesia was defined as three or more pain interventions, including epidural analgesia, NSAIDs, ketamine, acetaminophen and local anesthetic infiltration of the surgical site. Long acting opiate was either morphine or hydromorphone, expressed as morphine equivalents. Data is presented as median with interquartile range and as percentage or mean with 95\% confidence intervals. Student t-test and Fisher's exact test were used for comparison. Univariate analysis was performed on multimodal analgesia.

Results: The incidence of excessive pain in the post-anterior resection population was $19 \%$ [12\%-28\%]. Use of multi-modal analgesia was 20\% [13\%-29\%]. While excessive and controlled pain patients had comparable ASA and BMI, the excessive pain patients were 10 years younger [CI 4-16], with more chronic pain and substance abuse.

Multimodal pain management was slightly higher in excessive pain patients, but did not reach statistical significance for our sample size. Intraoperative morphine equivalents were similar. The most common pain adjunct was preoperative acetaminophen, followed by epidural anesthesia, ketamine, NSAIDs and lastly local infiltration.

Discussion: Multimodal pain management is currently underutilized in patients undergoing anterior resection. Intraoperative care had repercussions for PACU and ward, with the excessive pain patients requiring more rescue medications, having higher VAS scores, needing more time to achieve discharge pain criteria (VAS $\leq 4$ ) and taking longer to be discharged.

References: none 
Table 1: Pain Interventions \& Endpoints for Controlled and Excessive Pain Patients

\begin{tabular}{|c|c|c|c|c|c|}
\hline & $\begin{array}{c}\text { Controlled pain } \\
n=88\end{array}$ & $\begin{array}{c}\text { Missing } \\
\text { data }\end{array}$ & $\begin{array}{c}\text { Excessive pain } \\
\mathrm{n}=21\end{array}$ & $\begin{array}{c}\text { Missing } \\
\text { data }\end{array}$ \\
\hline \multirow{3}{*}{ Multimodal analgesia interventions } & $\leq 1$ & $42(48 \%)$ & & $9(43 \%)$ & \\
\hline & 2 & $29(33 \%)$ & & $7(33 \%)$ & \\
\hline & $\geq 3$ & $17(19 \%)$ & & $5(24 \%)$ & \\
\hline \multicolumn{2}{|l|}{ PACU morphine equivalents (mg) } & $5.4(0-12)$ & 1 & $19.4(9.7-26.5)$ & 1 \\
\hline \multicolumn{2}{|l|}{ PACU worst VAS score } & $5(2-7)$ & 17 & $8(7-9)$ & 1 \\
\hline \multicolumn{2}{|l|}{ Time to achieve pain control (min) } & $35(0-90)$ & 0 & $133(103-200)$ & 1 \\
\hline \multicolumn{2}{|l|}{ Length of PACU stay (min) } & $148(112-226)$ & 0 & $260(155-505)$ & 0 \\
\hline \multicolumn{2}{|c|}{$\begin{array}{l}\text { Ward morphine equivalents for patients } \\
\text { without epidurals }(\mathrm{mg} / 24 \mathrm{~h})\end{array}$} & $34(15-60)$ & $\begin{array}{c}1 \\
\mathrm{n}=57\end{array}$ & $80(49-135)$ & $\begin{array}{c}0 \\
\mathrm{n}=11\end{array}$ \\
\hline \multicolumn{2}{|l|}{ Ward worst VAS } & $4(2-6)$ & 1 & $6(5-9)$ & 1 \\
\hline
\end{tabular}

